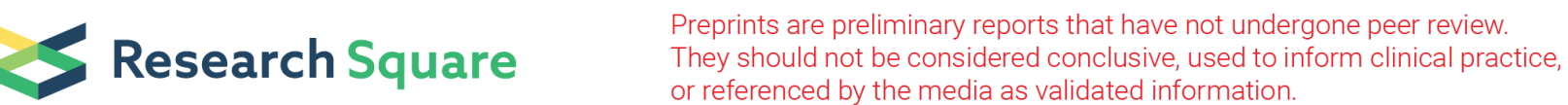

\section{A Method of Improving Physical Adsorption of Cellulase On Mesoporous Zr-Based MOF Surface: Enhanced Stability, Recyclability, And Catalytic Efficiency}

\section{YiWang (D 534507724@qq.com )}

JU: Jiangsu University https://orcid.org/0000-0002-9679-7012

\section{Juan Han}

Jiangsu University School of Food and Biological Engineering

Jia Cong Wu

Jiangsu University School of Chemistry and Chemical Engineering

Lei Wang

Jiangsu University School of Chemistry and Chemical Engineering

Chun Mei Li

Jiangsu University School of Chemistry and Chemical Engineering

Yang Zhou

Jiangsu University Institute of Life Sciences

Yun Wang

Jiangsu University School of Chemistry and Chemical Engineering

\section{Research Article}

Keywords: Cellulase, Biocatalyst, Cellulose degradation, Mesoporous metal organic framework

Posted Date: May 19th, 2021

DOI: https://doi.org/10.21203/rs.3.rs-482146/v1

License: (c) (i) This work is licensed under a Creative Commons Attribution 4.0 International License.

Read Full License 


\section{Abstract}

In this paper, we aimed at developing immobilized cellulase biocatalyst by enhancing the anchor of cellulase on support surface. A mesoporous Zr-based MOF was first synthesized by biomineralization method using dextran as template. The resultant PVP-cellulase@CD-UI0-66-Zr exhibited a high loading capacity of $265 \mathrm{mg} \mathrm{g}^{-1}$ support. The physical adsorption of cellulase on CD-UIO-66-Zr could be further enhanced by the capping of cellulase with PVP. About $83 \%$ of the activity of PVP-cellulase@CD-UIO-66-Zr could be retained after six cycles, and its equilibrium leakage ratio was $36 \%$ during thirty days' leaching test. It was noting that about $80 \%$ of activity of immobilized PVP-cellulase@CD-UIO-66-Zr could be retained after incubation at $80^{\circ} \mathrm{C}$ for 1 hour. The immobilized cellulase exhibited higher pH stability, thermostability, storage stability and catalytic efficiency than free one.

\section{Introduction}

The non-renewability of fossil energy and the increasingly prominent global environmental problems caused by it have made the development of biomass energy highly valued by governments of all countries(Danish and Wang 2019; Cao et al. 2020). Lignocellulose is the most widely distributed and abundant biomass resource on earth(Chandel et al. 2018; Karumuri et al. 2015). Through certain processing, cellulose can be transformed into a series of products with great application value, such as fuel(Ci et al. 2017), chemical raw materials(Ji et al. 2019) and pharmaceutical products(Zhang et al. 2015a). The utilization and transformation of cellulose is of great significance to solve the world energy crisis and environmental pollution problems.

Cellulose is a linear polymer compound bound by glucose molecules with $\beta-1,4$-glycosidic bonds, and generally requires pretreatment to be degraded into small molecular sugars before it can be used. Lignocellulose can be degraded by physical method(Chen et al. 2017b), chemical method(Zhang et al. 2020), biological method(Wang et al. 2020). Biological method generally uses cellulase to hydrolyze lignocellulose into glucose. It has the advantages of mild conditions, environmental friendliness, strong specificity, high catalytic efficiency, and low unit energy consumption, meeting the sustainable development of today's society demand. However, the use cost of cellulase accounts for $50 \%$ of the total cost of cellulose enzymatic hydrolysis and saccharification(Gokhale and Lee 2012). Therefore, obtaining immobilized enzymes with more stable performance and recyclable utilization is an effective way to reduce the application cost of cellulase.

The catalytic performance of immobilized enzymes is highly dependent on the property of carrier materials(Drout et al. 2019; Hu et al. 2018). The desired carrier materials should have the characteristics of good stability, easy regulation of physical and chemical properties. A series of carrier materials, such as natural products(Wang et al. 2016; Aigner and Scheibel 2019), inorganic carriers(Grewal et al. 2017; Zhang et al. 2019), organic polymers(Lozano et al. 2014), magnetic carriers(Hosseini et al. 2018; Zhou et al. 2020), and metal organic framework(Qi et al. 2018; Phipps et al. 2020), have been developed for enzyme immobilization. As a new type of porous material, metal-organic framework is a kind of porous 
coordination polymer with two-dimensional or three-dimensional pore structure connected by metal nodes and organic ligands through coordination bonds(Cui et al. 2018). MOF materials have the advantages of high porosity and high specific surface, so its application is in full swing, especially in the field of enzyme immobilization(Liang et al. 2015).

There are relatively few studies on the use of mesoporous MOF materials for the immobilization of cellulase. The diffusion of macromolecular cellulose into micro/mesoporous MOF is often limited. The currently reported immobilization of cellulase on MOF is mainly surface adsorption(Khoshnevisan et al. 2011) and covalent binding(Huang et al. 2020). Multi-step reactions are often involved in covalent bonding process, and the preparation of carrier materials is complicated. The physical adsorption method has remarkable characteristics of simple and mild immobilization process, reducing the loss of activity during immobilization process. However, the immobilized enzyme is easily detached from the carrier material due to weak binding force, which is not conducive to the recycling of enzyme.

UIO-66-Zr is a MOF material with highly stable secondary building units $\mathrm{Zr}_{6} \mathrm{O}_{4}(\mathrm{OH})_{4}$, which makes $\mathrm{UIO}-66$ Zr highly chemically and thermally stable(Schaate et al. 2011; Decoste et al. 2013). Lipase was immobilized on UIO-66-Zr by surface adsorption with $202.4 \mathrm{mg} \mathrm{g}^{-1}$ loading capacity(Liu et al. 2015). Soybean epoxy-hydrolase (SEH) can be effectively fixed on the amino surface of UIO-66- $\mathrm{NH}_{2} \mathrm{MOF}$ by cross-linking(Cao et al. 2016). To the best of our knowledge, the immobilization of cellulase onto bare UIO-66-Zr has seldom been reported. Meanwhile, the synthesis of nanocrystals of mesoporous UiO-66-Zr for enzyme immobilization is not straightforward. Although the $\mathrm{NH}_{2}$ functionalized UIO-66-Zr has been developed as carrier for anchoring cellulase(Ahmed et al. 2018; Zhou et al. 2019), the adsorption mechanism of cellulase onto UIO-66-Zr and how to enhance adsorption needs to be further explored.

In this paper, a mesoporous UIO-66-Zr was first synthesized by biomineralization method using dextran as template, and the prepared MOF was used as support material for cellulase immobilization by physical adsorption. Furthermore, a small amount of PVP was used to improve the stability of immobilized cellulase. The resultant mesoporous MOF before and after enzyme immobilization were characterized by BET, FT-IR, XRD, TGA, DLS, SEM and TEM. The stability, reusability, and catalytic efficiency of immobilized cellulase were investigated.

\section{Materials And Methods}

\subsection{Materials}

Zirconium chloride ( $\left.\mathrm{ZrCl}_{4}, 98 \%\right), \mathrm{N}, \mathrm{N}$-dimethylformamide (DMF, 98\%), hydroxymethyl cellulose sodium (CMC, 98\%), acetic acid (98\%), sodium acetate (98\%), 3,5-dinitrosalicylic acid (98\%), sodium hydroxide $(98 \%)$, potassium sodium tartrate (98\%), phenol (98\%), polyvinylpyrrolidone $(98 \%)$ and sodium sulfite anhydrous (98\%) were purchased from Sinopharm Chemical Reagent Co. Ltd (Shanghai, China). Cellulase from Trichoderma reesei ATCC 26921 ( $\geq 45$ units $/ \mathrm{mg}$ ) was purchased from Aladdin. Dextran1500 was purchased from Energy Chemical (Shanghai, China). Bicinchoninic acid (BCA) Protein 
Assay Kit and bovine serum albumin (BSA) were obtained from Thermo Scientific Co., Ltd (China). The water used throughout the experiments was deionized. All chemicals were used as received without further treatment.

\subsection{Preparation of UI0-66-Zr, Dex@UI0-66-Zr and CD-UIO- $66-\mathrm{Zr}$}

The preparation of the UIO-66-Zr was based on the reported method with some modification(Huang et al. 2015). $\mathrm{ZrCl}_{4}(0.7 \mathrm{mmol})$ and $\mathrm{BDC}(0.7 \mathrm{mmol})$ were first dissolved in $10 \mathrm{~mL} \mathrm{DMF}$, then the suspension solution was sonicated for 30 minutes followed by the incubation in an oil bath at $80{ }^{\circ} \mathrm{C}$ for $24 \mathrm{~h}$. The solid products were collected by centrifugation and washed three times with DMF and methanol to remove the residual reactant. The resultant samples were dried at $60{ }^{\circ} \mathrm{C}$ overnight.

The preparation of Dex@UI0-66-Zr was conducted by dissolving $\mathrm{ZrCl}_{4}(0.7 \mathrm{mmol}), \mathrm{BDC}(0.7 \mathrm{mmol})$, and Dextran 1500 (2 mg) into $10 \mathrm{~mL}$ DMF respectively. The following steps were same as that of UIO-66-Zr. The prepared Dex@Ul0-66-Zr was then placed in a tube furnace and calcined at $325^{\circ} \mathrm{C}$ for 2 hours. The resultant sample was activated in $10 \mathrm{~mL}$ of methanol for three days to obtain CD-UIO-66-Zr.

\subsection{Preparation of cellulase@UI0-66-Zr, cellulase@CD-UIO- 66-Zr, PVP-cellulase@CD-UI0-66-Zr}

The immobilized cellulase, including cellulase@UI0-66-Zr and cellulase@CD-UIO-66-Zr, were prepared by dissolving $10 \mathrm{mg}$ substrate in $2.5 \mathrm{~mL}$ of acetic acid including cellulase. The preparation of PVPcellulase@CD-UIO-66-Zr were conducted by dissolving 10 mg CD-UIO-66-Zr in 2.5 mL of 0.5\% PVP including cellulase. The reaction was conducted at room temperature with stirring for $12 \mathrm{~h}$, then the solids were separated by centrifugation at $6000 \mathrm{rpm}$ for $10 \mathrm{~min}$. The product was washed by deionized water and dried in vacuum freeze dryer for $10 \mathrm{~h}$, eventually saved at $4^{\circ} \mathrm{C}$.

\subsection{Characterization of support materials and immobilized cellulase}

Fourier transform infrared (FT-IR) spectra were collected in the range $3500-500 \mathrm{~cm}^{-1}$ on a Nicolet Nexus 470 Spectrometer. $\mathrm{N}_{2}$ adsorption-desorption isotherms and pore size distribution were measured by a Brunauer-Emmett-Teller (BET) instrument (ASAP 3020, Micromeritics). Before BET measurement, all samples were degassed at $150^{\circ} \mathrm{C}$ under reduced pressure overnight. Powder X-ray diffraction (XRD) patterns were recorded by an X-ray diffractometer (XRD-6100Lab, Japan) with scattering angles (20) of $5-40^{\circ} \mathrm{C}$ at a scan rate of $7^{\circ} \mathrm{C} \mathrm{min}^{-1}$. Thermal gravimetric analysis (TGA) was performed using the STA449C Thermal Analyzer. The dried sample was filled into a crucible, and the TGA curve was collected from 10 to $1000^{\circ} \mathrm{C}$ with a heating rate of $10^{\circ} \mathrm{C} \mathrm{min}{ }^{-1}$ under a continuous stream of nitrogen gas. The morphologies of all samples were recorded by JSM-6010 PLUS/LA scanning electron microscope (SEM) and FEI Tecnai G2 S-TWIN F20 transmission electron microscope (TEM). DLS were recorded by particle size analyzer (Mastersizer 3000, Malvern Instruments Ltd., UK). 


\subsection{Enzyme activity assay}

The activity of cellulase was assessed by incubating cellulase with the $\mathrm{CMC}$ substrate for $30 \mathrm{~min}$ at $50^{\circ} \mathrm{C}$ in a $0.2 \mathrm{M}$ acetic buffer ( $\mathrm{pH}$ 5.0). The amount of produced glucose was examined using dinitrosalicylic acid method and the absorbance was measured at $540 \mathrm{~nm}$ (Miller et al. 1960). The concentration of enzyme was measured by PierceTM BCA protein assay kit. The Loading efficiency and Loading capacity were calculated according to Eq. 1 and Eq. 2 respectively.

Loading efficiency $(\%)=\frac{m-c_{1} v_{1}}{m} \times 100 \%$

Loading capacity $\left(\mathrm{mg} \mathrm{g}^{-1}\right)=\frac{m-c_{1} v_{1}}{M}$

Where $m(\mathrm{mg})$ is the mass of cellulase initially added to the solution; $C_{1}\left(\mathrm{mg} \mathrm{mL}^{-1}\right)$ is the concentration of cellulase in supernatant; $V_{1}(\mathrm{~mL})$ is the volume of supernatant; $M(\mathrm{~g})$ is the weight of the immobilized cellulase biocomposite.

\subsection{Stability and reusability of immobilized cellulase}

The $\mathrm{pH}$ stability and thermal stability were examined by incubating enzyme solution at various $\mathrm{pH}$ values $(3.0-8.0)$ and temperatures $\left(30-80^{\circ} \mathrm{C}\right)$ for 60 min respectively with other conditions at the optimum. To analyze the reusability of immobilized cellulase, the biocatalyst was recovered by centrifugation and washing with deionized water for the next test. The reaction was repeated 8 times and the initial activity was set as $100 \%$. The storage stabilities of free and immobilized cellulase were examined for 30 days with an interval of three days at $4{ }^{\circ} \mathrm{C}$. The leaching test was conducted as the storage stability test except that the biocatalyst was incubated in solution. The initial activity was regarded as $100 \%$.

\subsection{Kinetic parameters}

The initial reaction rates of free and immobilized cellulase for catalytic conversion of different concentrations of substrates were measured, and the data were plotted using a double-reciprocal Lineweaver-Burk equation. The apparent kinetics parameters $\left(K_{\mathrm{m}}\right.$ and $\left.V_{\max }\right)$ were calculated using Eq. 3.

$\frac{1}{V}=\frac{K_{m}}{V_{m}} \times \frac{1}{[S]}+\frac{1}{V_{m}}$

Where $V$ represents the initial reaction rate $\left(\mathrm{mg} \mathrm{mL}^{-1} \mathrm{~min}^{-1}\right) ; V_{\mathrm{m}}$ represents the maximum reaction rate $\left(\mathrm{mg} \mathrm{mL}^{-1} \mathrm{~min}^{-1}\right)$; $[S]$ is the initial substrate concentration $\left(\mathrm{mg} \mathrm{mL}^{-1}\right) ; K_{\mathrm{m}}$ represents the Michaelis-Menten constant $\left(\mathrm{mg} \mathrm{mL}^{-1}\right)$.

\subsection{Fluorescent Labeling of cellulase}


First, $10 \mathrm{mg}$ cellulase was weighed and dissolved in $5 \mathrm{~mL}$ acetate buffer solution, then $5 \mathrm{mg}$ carbodiimide hydrochloride (EDC) and $2.5 \mathrm{mg} \mathrm{N}$-hydroxysuccinimide (NHS) were added. After the reaction was lasted for $1 \mathrm{~h}$ at $4^{\circ} \mathrm{C}, 100 \mathrm{ug}$ of fluorescein isothiocyanate (FITC) was added to the solution, and the reaction was lasted for 4 hours at low temperature $\left(4^{\circ} \mathrm{C}\right)$ in dark. To obtain the fluorescently labeled cellulase (FITC-cellulase), the solution was dialyzed one day to remove unreacted EDC, NHS and FITC.

\section{Results And Discussion}

\subsection{Fabrication and Characterization of stable zirconium based meso-MOF}

In this paper, two kinds of UiO-66-Zr were prepared for the immobilization of cellulase. The conventional UiO-66-Zr was synthesized by hydrothermal synthesis in Teflon liner vessel for $24 \mathrm{~h}$ under high temperature, such as $120^{\circ} \mathrm{C}$ and $150^{\circ} \mathrm{C}$ (Hendrickx et al. 2015; Huang et al. 2015). Here, we prepared UiO$66-\mathrm{Zr}$ at a relatively low temperature of $80^{\circ} \mathrm{C}$ for $24 \mathrm{~h}$. As a typical polysaccharide, dextran 1500 was used as template for the fabrication of mesoporous UiO-66-Zr. As shown in Scheme 1, the synthesis process can be primarily divided into the following steps: (a) Dextran1500@UI0-66-Zr (Dex@UI0-66-Zr)was prepared through biomineralization process by mixing aqueous solutions of $\mathrm{ZrCl}_{4}, \mathrm{BTC}$ and Dex1500 under mild conditions; (b) Dex1500 was then removed through high-temperature calcination to form the solid carrier, named as CD-UIO-66-Zr. The immobilized cellulase (cellulase@CD-UIO-66-Zr and PVPcellulase@CD-UiO-66-Zr) were synthesize by the adsorption of cellulase onto solid carrier.

The FTIR spectrum was analyzed to confirm the composition of as-prepared MOF (Fig. 1. (a)). The vibration peak at $746 \mathrm{~cm}^{-1}$ was ascribed to the $\mathrm{Zr}-\mathrm{O}$ bond of UIO-66. The carboxyl group existing in the terephthalic acid linker of UIO-66-Zr can be proved by the characteristic peak at $1660 \mathrm{~cm}^{-1}$, which was the vibration absorption of the $\mathrm{C}=0$ double bond in carboxyl group, while $1585 \mathrm{~cm}^{-1}$ was the absorption peak caused by the stretching vibration of carboxyl(Kandiah et al. 2010). The successful formation of Dex@UI0-66-Zr can be proved because the characteristic C-0 bond of dextran1500 appeared at $1158 \mathrm{~cm}$ ${ }^{-1}$ (Fathi et al. 2011), and the disappear of the characteristic peak indicated the remove of dextran 1500 after calcination.

The XRD patterns of Zr-based MOF materials were shown in Fig. 1 (b). All diffraction peaks of the prepared MOFs matched well with the simulated standard patterns of UIO-66-Zr. The incorporation of dextran 1500 had no significant influence on the crystallinity of UIO-66-Zr. The decrease in the diffraction intensity of CD-UIO-66-Zr indicated that the high temperature calcination process to move dextran 1500 in Dex@UI0-66-Zr might reduce crystallinity or generate numerous defects in the architecture of CD-UIO-66Zr(Shearer et al. 2014).

TGA spectrum was shown in Fig. 1 (c). Dextran 1500 has a two-stage thermal decomposition process: the first weight loss $\left(<200^{\circ} \mathrm{C}\right)$ was due to the loss of free water and bound water; the second weight loss was because of the structure collapse of dextran 1500 . So the calcining temperature for removing 
dextran 1500 was set at $325^{\circ} \mathrm{C}$ by TGA curve of dextran 1500 . It can be deduced from the TGA curve of CD-UIO-66-Zr that its structure collapsed within the temperature range of $500-600^{\circ} \mathrm{C}$.

The morphologies of Zr-based MOF were characterized by SEM and TEM, and uniform and aggregated MOF particles could be observed in Fig. 2 and Fig. S1. Compared with conventional UIO-66-Zr, the resultant Dex@UI0-66-Zr and CD-UIO-66-Zr showed an increase in aggregation. The average particle size of UIO-66-Zr determined by dynamic light scattering was $179.8 \mathrm{~nm}$, while the particle size of Dex@UIO-66$\mathrm{Zr}$ and CD-UIO-66-Zr increased to $389.9 \mathrm{~nm}$ and $497.8 \mathrm{~nm}$ because of aggregation (Fig. 2).

The pore size and distribution of the resultant Zr-based MOF particles was analyzed by $\mathrm{N}_{2}$ adsorption desorption (Fig. 3). The hysteresis loops at $\mathrm{P} / \mathrm{P}_{0}$ value around 0.4 and 0.8 in the isotherms confirmed the existence of mesopores in the prepared Zr-based MOF particles. The adsorption-desorption isotherms of UIO-66-Zr and CD-UIO-66-Zr possessed a type $\otimes$ curve with $\mathrm{H} 1$ hysteresis loop(Sing et al. 1985). The successful preparation of mesoporous UiO-66-Zr can also be demonstrated by the BJH pore diameter distributions (Fig. 3(a)). As shown in Table 1, the specific surface area and total pore volume of mesoporous CD-UIO-66-Zr are $646.9 \mathrm{~m}^{2} \mathrm{~g}^{-1}$ and $0.2809 \mathrm{~cm}^{3} \mathrm{~g}^{-1}$ respectively, which are slightly low than UIO-66-Zr. However, the average pore size of CD-UIO-66-Zr increased from $2.255 \mathrm{~nm}$ to $4.617 \mathrm{~nm}$. Polysaccharides have been used to induced biomineralization of ZIF-8, resulting in the formation of protective shell around polysaccharides(Liang et al. 2017). The introduction of dextran 1500 as template for preparing UIO-66-Zr played a key role in the fabrication of mesoporous UIO-66-Zr.

Table 1

BET surface area, and pore size data of Zr-based MOF particles

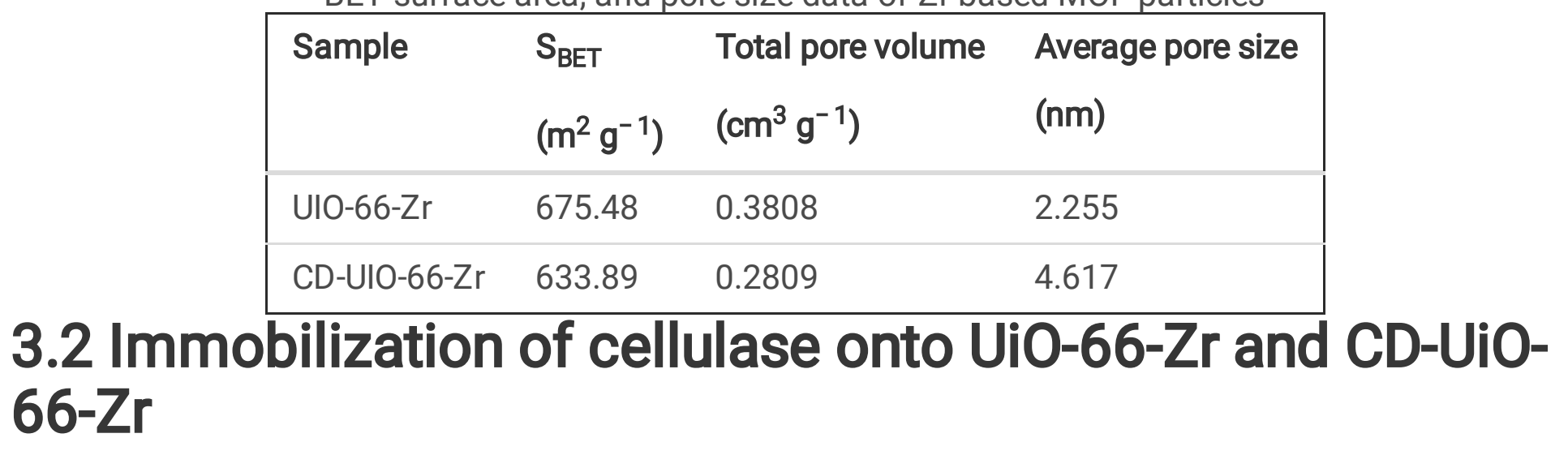

The prepared UiO-66-Zr and CD-UiO-66-Zr with narrow pore distribution around 2.255 and $4.617 \mathrm{~nm}$ were used as carrier for anchoring cellulase. Cellulase has been successfully immobilized on UIO-66-Zr with a loading capacity of $102 \mathrm{mg} \mathrm{g}^{-1}$.(Zhou et al. 2019) As shown in Fig. 4(a), the maximum loading capacity around $310 \mathrm{mg} \mathrm{g}^{-1}$ could be both achieved for the resultant UiO-66-Zr and CD-UiO-66-Zr at cellulase concentration of $2 \mathrm{mg} \mathrm{mL}^{-1}$ in HAc-NaAc buffer system, and the loading capacity of PVP-cellulase@CDUiO-66-Zr slightly decreased to $265 \mathrm{mg} \mathrm{g}^{-1}$. As can be seen from the thermogravimetry curves in Fig. 1 (c), the weight loss of second stage for PVP-cellulase@CD-U0-66-Zr and cellulase@CD-U0-66-Zr induced by the decomposition of cellulase were $20 \%$ and $24 \%$ respectively, which were basically consistent with 
the loading capacity of PVP-cellulase@CD-U0-66-Zr $\left(265 \mathrm{mg} \mathrm{g}^{-1}\right)$ and cellulase@CD-UO-66-Zr (312 mg $\mathrm{g}^{-1}$ ) determined by the BCA kit.

Polyvinylpyrrolidone (PVP) has been employed as a capping agent for the stabilization of cellulase(Lyu et al. 2014; Liang et al. 2016). The physical adsorption of cellulase onto UIO-66-Zr and CD-UIO-66-Zr was performed by incubating the solid supports in a solution containing PVP-modified cellulase. The resulting PVP-cellulase@CD-UIO-66-Zr and PVP-cellulase@UI0-66-Zr showed an obvious increase in recycling stability compared to corresponding cellulase@CD-UI0-66-Zr and cellulase@UIO-66-Zr, and about 83\% of the activity of PVP-modified cellulase anchoring on CD-UIO-66-Zr could be retained after 6 cycles, while that of cellulase@UIO-66-Zr, cellulase@CD-UIO-66-Zr, and PVP-cellulase@UIO-66-Zr dropped to 17\%, 47\% and $38 \%$ respectively (Fig. $4 \mathrm{~b}$ ). Some reported cycling stability of cellulase immobilized on various solid supports were summarized in Table 2. The adsorption of PVP-capping cellulase onto meso-CD-UIO-66-Zr exhibited enhanced recycling stability relative to other reported systems.

Table 2

Cycling stability of cellulase immobilized on various carriers

\begin{tabular}{|lll|}
\hline Supports & Cycles & Residual activity \\
\hline CD-UiO-66-Zr(PVP) & 5 & $88 \%$ (This work) \\
\hline MNPs-APTES-Cu & 5 & $73 \%$ (Abbaszadeh and Hejazi 2019) \\
\hline $\mathrm{MnO}_{2}$ & 5 & $60 \%($ Cherian et al. 2015) \\
\hline Biochar & 5 & $75 \%($ Zhu et al. 2018) \\
\hline Ch-Ce & 5 & $50 \%($ Saha et al. 2019) \\
\hline AMNPs & 7 & $54 \%($ Ladole et al. 2017) \\
\hline
\end{tabular}

Then, a leaching test was conducted by incubating the immobilized cellulase in buffer solution for 30 days.. As shown in Fig. 4c, the leakage ratio of immobilized cellulase gradually increased and be to in equilibrium after 21 days, and that of PVP-cellulase@CD-UIO-66-Zr was 32\%. In general, the leakage ratio of PVP-cellulase@CD-UIO-66-Zr remained lower than the other immobilized enzyme throughout the entire incubating period, which indicated that the detachment of cellulase from CD-UIO-66-Zr could be greatly inhibited once the cellulase molecules was modified with PVP. So, the capping of cellulase by PVP, to some extent, enhance the anchoring of cellulase on the surface pore of UIO-66-Zr, which contributes to the improvement in recyclability compared with cellulase@CD-UIO-66-Zr.

It was an interesting phenomenon that CD-UIO-66-Zr exhibited higher adsorption strength with cellulase than UIO-66-Zr. Since the size of cellulase $(12.4 \mathrm{~nm} \times 3.7 \mathrm{~nm}$ )(Chen et al. 2017a) was much larger than the pore size of UIO-66-Zr and CD-UIO-66-Zr, it's difficult for cellulase to diffuse into pore through specific orientation. The dye-tagging strategy has been developed to enhance adsorption of proteins onto MOF with smaller pore size than protein molecules(Mehta et al. 2016). It was achieved by the conjugation of 
small fluorescein isothiocyanate (FITC) dye molecules with trypsin. Although the adsorption of large molecules into the pore of MOF was hindered, the small FITC molecules $\left(0.9 \times 1.2 \times 1.4 \mathrm{~nm}^{3}\right)$ could enter the pores and enhance the anchoring of large biomolecules on MOF. We prepared FITC-cellulase conjugate and studied the adsorption of FITC-cellulase on UIO-66-Zr and CD-UIO-66-Zr. The penetration of FITC molecules into the pore of MOF was confirmed by laser scanning confocal microscopy (CLSM) characterization (Fig. 5a). Owing to slightly larger pore size of CD-UIO-66-Zr (4.617 nm) than the corresponding UIO-66-Zr $(2.255 \mathrm{~nm})$, as displayed in Scheme 1, the cellulase molecules like a long stick and the short axis $(3.7 \mathrm{~nm})$ make the stick could only partially plug into the pore of CD-UIO-66-Zr, and the adsorption of cellulase on CD-UIO-66-Zr can be enhanced. We also analyzed the BJH $\mathrm{N}_{2}$ adsorptiondesorption isotherms of CD-UO-66-Zr after the immobilization of cellulase (Fig. 5b). Compared with CDU0-66-Zr $\left(633 \mathrm{~m}^{2} \mathrm{~g}^{-1}\right)$, the decrease in the specific surface of PVP-cellulase@CD-UO-66-Zr $\left(499 \mathrm{~m}^{2} \mathrm{~g}^{-1}\right)$ was not significant and the mesoporous structure was preserved, which may be due to the partial plugging of PVP-cellulase into the pore of CD-UIO-66-Zr.

The structure of the immobilized cellulase@CD-UIO-66-Zr were analyzed by FT-IR and XRD. Figure 1 (a) showed the FT-IR spectrum of cellulase@CD-UIO-66-Zr and PVP-cellulase@CD-UIO-66-Zr. The weak bands revealed at 1620 and $1028 \mathrm{~cm}^{-1}$ were ascribed to the $\mathrm{C}-\mathrm{N}$ stretching vibration of cellulase, which demonstrated the successful immobilization of cellulase. As displayed in Fig. 1(b), the adsorption of cellulase on CD-UIO-66-Zr had no obvious effect on the position and intensity of diffraction peaks, indicating that the structure of CD-UIO-66-Zr was well retained during enzyme immobilization. It can be clearly seen from the SEM image in Fig. 2 that the anchoring of cellulase on the surface of Zr-based MOF carrier had no significant influence on the monography of CD-UIO-66-Zr.

\subsection{Stability of free cellulase and immobilized cellulase}

It is well known that the catalytic activity of free cellulase and cellulase@CD-UIO-66- $\mathrm{Zr}$ is dependent on reaction conditions. Enzymes exhibit their maximum activity at optimum solution $\mathrm{pH}$ and reaction temperature. As shown in Fig. 6, the free cellulase showed the highest activity at $\mathrm{pH} 5$, while the maximum activity for cellulase@CD-UIO-66-Zr and PVP-cellulase@CD-UI0-66-Zr was achieved in a pH range of 5 to 6. . The effect of temperature from $30^{\circ} \mathrm{C}$ to $80^{\circ} \mathrm{C}$ on the activities of free and immobilized cellulase was investigated at $\mathrm{pH}$ 5.0. The optimum temperature of free cellulase, cellulase@CD-UIO-66-Zr and PVP-cellulase@CD-UIO-66-Zr are $50^{\circ} \mathrm{C}, 60^{\circ} \mathrm{C}$ and $60-70^{\circ} \mathrm{C}$ respectively.

The immobilized cellulase exhibited enhanced $\mathrm{pH}$, thermal and storage stabilities compared with free cellulase and previous immobilized cellulase(Xu et al. 2014; Zhang et al. 2015b). It was noting that after incubation at pH 8.0 for 1 hour, as shown in Fig. 7(a), the activity loss of free cellulase was $56 \%$, while the immobilized cellulase@CD-UIO-66-Zr and PVP-cellulase@CD-UIO-66-Zr maintained 77\% and 80\% of its original activity, respectively. Meanwhile, the thermal stabilities were analyzed by incubating the free and immobilized cellulase at different temperatures for 1 hour. As shown in Fig. 7(b), when rising temperature beyond $70^{\circ} \mathrm{C}$, a drastic activity loss was observed for free enzyme. The activity loss of free cellulase was $51 \%$ after incubation at $80^{\circ} \mathrm{C}$ for 1 hour, while $82 \%$ and $85 \%$ of original activity were preserved for cellulase@CD-UI0-66-Zr and PVP-cellulase@CD-UIO-66-Zr respectively. The immobilized cellulase on CD- 
UI0-66-Zr exhibited higher thermal tolerance than previously reported cellulase immobilization system(Abraham et al. 2014). The storage stabilities were analyzed by storing free and immobilized cellulase at $4^{\circ} \mathrm{C}$ for 30 days. As shown in Fig. 7 (c), with the extension of storage time, free cellulase retained only $15 \%$ of its original activity after 30 days, while $50 \%$ and $63 \%$ were preserved for cellulase@CD-UIO-66-Zr and PVP-cellulase@CD-UIO-66-Zr respectively.

These results indicated that the immobilization of cellulase on CD-UIO-66-Zr greatly improved the $\mathrm{pH}$, thermal, storage and recycling stabilities of cellulase. Meanwhile, the modification of cellulase with PVP effectively improve the stability especially recycling stability relative to cellulase@CD-UIO-66-Zr by enhancing the anchoring of cellulase on the mesoporous CD-UIO-66-Zr.

\subsection{Catalytic kinetics of free cellulase and immobilized cellulase}

The catalytic kinetics of cellulase@CD-UIO-66-Zr and PVP-cellulase@CD-UIO-66-Zr were also analyzed by Michaelis-Menten equation. The Lineweaver-Burk plots were displayed in Fig. S3, and the kinetic parameters were listed in Table 3. Compared with free cellulase, the $K_{\mathrm{m}}$ value of cellulase@CD-UIO-66-Zr and PVP-cellulase@CD-UIO-66-Zr slightly decreased to $13.387 \mathrm{mg} \mathrm{mL}^{-1}$ and $13.355 \mathrm{mg} \mathrm{mL}^{-1}$ respectively, demonstrating the full contact of $\mathrm{CMC}$ to the active sites of immobilized cellulase(Hosseini et al. 2018). The $K_{\text {cat }} / K_{\mathrm{m}}$ values of PVP-cellulase@CD-UIO-66-Zr $\left(1.311 \mathrm{~mL} \mathrm{mg}^{-1} \mathrm{~min}^{-1}\right)$ was slightly higher than free one $\left(0.732 \mathrm{~mL} \mathrm{mg}^{-1} \mathrm{~min}^{-1}\right)$, which indicated that a high catalytic efficiency was achieved for PVP-cellulase@CD-UIO-66-Zr relative to free cellulase.

Table 3

Kinetic parameters of free and immobilized cellulase.

\begin{tabular}{|c|c|c|c|c|}
\hline Kinetic parameters & $\begin{array}{l}K_{\mathrm{m}} \\
\left(\mathrm{mg} \mathrm{mL}^{-1}\right)\end{array}$ & $\begin{array}{l}K_{\text {cat }} \\
\left(\min ^{-1}\right)\end{array}$ & $\begin{array}{l}V_{\mathrm{m}} \\
\left(\mathrm{mg} \mathrm{mL}^{-1} \mathrm{~min}^{-1}\right)\end{array}$ & $\begin{array}{l}K_{\text {cat }} / K_{\mathrm{m}} \\
\left(\mathrm{mL} \mathrm{mg}{ }^{-1} \mathrm{~min}^{-1}\right)\end{array}$ \\
\hline Free cellulase & 14.525 & 10.63 & 1.063 & 0.732 \\
\hline cellulase@CD-UIO-66-Zr & 13.387 & 12.85 & 1.285 & 0.959 \\
\hline PVP-cellulase@CD-UIO-66-Zr & 13.355 & 17.51 & 1.751 & 1.311 \\
\hline
\end{tabular}

\section{Conclusion}

In this study, a mesoporous Zr-based MOF was prepared by biomineralization method using dextran as template, and the resultant CD-UIO-66-Zr was successfully used for the surface adsorption of cellulase with a high loading capacity about $265 \mathrm{mg} \mathrm{g}^{-1}$. The better recycling stability of CD-UIO-66-Zr could be due to the partial inserting of cellulase into its surface pore. The capping of cellulase with PVP is conductive to enhance the anchoring of cellulase on CD-UIO-66-Zr. The successful preparation of Zr-based MOF with 
moderate pore size shows the promising future of using MOF as carrier for cellulase immobilization by simple surface adsorption method.

\section{Declarations}

\section{Acknowledgment}

We gratefully acknowledge the National Natural Science Foundation of China (Nos. 21878131, 22078133 and 21676124) for the financial support.

The authors declare no competing financial interest and no animal or human participants were involved in the study.

\section{References}

1. Abbaszadeh $\mathrm{M}$, Hejazi $\mathrm{P}$ (2019) Metal affinity immobilization of cellulase on $\mathrm{Fe}_{3} \mathrm{O}_{4}$ nanoparticles with copper as ligand for biocatalytic applications. Food Chem 290:47-55

2. Abraham R, Verma M, Barrow C, Puri M (2014) Suitability of magnetic nanoparticle immobilised cellulases in enhancing enzymatic saccharification of pretreated hemp biomass. Biotechnol Biofuels $7: 2-12$

3. Ahmed I, Yang X, Dubale A, Li R-F, Ma Y-M, Wang L-M, Hou G-H, Guan R-F, Xie M-H (2018) Hydrolysis of cellulose using cellulase physically immobilized on highly stable zirconium based metal-organic frameworks. Bioresour Technol 270:377-382

4. Aigner T, Scheibel T (2019) Self-Rolling Refillable Tubular Enzyme Containers Made of Recombinant Spider Silk and Chitosan. ACS Appl Mater Interfaces 11:15290-15297

5. Cao Q, Zhu M, Chen J, Song Y (2020) Novel Lignin-Cellulose-Based Carbon Nanofibers as HighPerformance Supercapacitors. ACS Appl Mater Interfaces 12:1210-1221

6. Cao S, Yue D, Li X, Smith T, Li N, Zong M, Wu H, Ma Y, Lou W (2016) Novel Nano-/Micro-Biocatalyst: Soybean Epoxide Hydrolase Immobilized on UiO-66- $\mathrm{NH}_{2}$ MOF for Efficient Biosynthesis of Enantiopure (R)-1, 2-Octanediol in Deep Eutectic Solvents. ACS Sustain Chem Eng 4:3586-3595

7. Chandel A, Garlapati V, Singh A, Antunes F, Silva S (2018) The path forward for lignocellulose biorefineries: Bottlenecks, solutions, and perspective on commercialization. Bioresour Technol 264:370-381

8. Chen B, Qiu J, Mo H, Yu Y (2017a) Synthesis of mesoporous silica with different pore sizes for cellulase immobilization: Pure physical adsorption. New J Chem 41:9338-9345

9. Chen H, Liu J, Chang X, Chen D, Xue Y, Liu P, Lin H, Han S (2017b) A review on the pretreatment of lignocellulose for high-value chemicals. Fuel Process Technol 160:196-206

10. Cherian E, Dharmendirakumar M, Baskar G (2015) Immobilization of cellulase onto $\mathrm{MnO}_{2}$ nanoparticles for bioethanol production by enhanced hydrolysis of agricultural waste. Chinese $\mathrm{J}$ 
Catal 36:1223-1229

11. Ci J, Chun C, Kuga S, Shen J (2017) Improved Performance of Microbial Fuel Cell Using Esterified Corncob Cellulose Nanofibers to Fabricate Air-Cathode Gas Diffusion Layer. ACS Sustain Chem Eng 5:9614-9618

12. Cui J, Ren S, Sun B, Jia S (2018) Optimization protocols and improved strategies for metal-organic frameworks for immobilizing enzymes: Current development and future challenges. Coord Chem Rev 370:22-41

13. Danish, Wang Z (2019) Does biomass energy consumption help to control environmental pollution? Evidence from BRICS countries. Sci Total Environ 670:1075-1083

14. Decoste J, Peterson G, Jasuja H, Glover T (2013) Stability and degradation mechanisms of metalorganic frameworks containing the $\mathrm{Zr}_{6} \mathrm{O}_{4}(\mathrm{OH})_{4}$ secondary building unit. J Mater Chem A 1:56425650

15. Drout R, Robison L, Farha O (2019) Catalytic applications of enzymes encapsulated in metalorganic frameworks. Coord Chem Rev 381:151-160

16. Fathi E, Atyabi N, Imani M, Alineiad Z (2011) Physically crosslinked polyvinyl alcohol-dextran blend xerogels: Morphology and thermal behavior. Carbohydr Polym 84:145-152

17. Gokhale AA, Lee I (2012) Cellulase immobilized nanostructured supports for efficient saccharification of cellulosic substrates. Top Catal 55:1231-1246

18. Grewal J, Ahmad R, Khare SK (2017) Development of cellulase-nanoconjugates with enhanced ionic liquid and thermal stability for in situ lignocellulose saccharification. Bioresour Technol 242:236243

19. Hendrickx K, Vanpoucke DEP, Leus K, Lejaeghere K, Hemelsost K (2015) Understanding Intrinsic Light Absorption Properties of UiO-66 Frameworks: A Combined Theoretical and Experimental Study. Inorg Chem 54:10701-10710

20. Hosseini S, Hosseini S, Zohreh N, Yaghoubi M, Pourjavadi A (2018) Covalent Immobilization of Cellulase Using Magnetic Poly(ionic liquid) Support: Improvement of the Enzyme Activity and Stability. J Agric Food Chem 66:789-798

21. Hu Y, Dai L, Liu D, Du W, Wang Y (2018) Progress \& prospect of metal-organic frameworks (MOFs) for enzyme immobilization (enzyme/MOFs). Renew Sustain Energy Rev 91:793-801

22. Huang H, Li J, Wang K, Han T, Zhong C (2015) An in situ self-assembly template strategy for the preparation of hierarchical-pore metal-organic frameworks. Nat Commun 6:1-8

23. Huang W, Pan S, Li Y, Yu L, Liu R (2020) Immobilization and characterization of cellulase on hydroxy and aldehyde functionalized magnetic $\mathrm{Fe}_{2} \mathrm{O}_{3} / \mathrm{Fe}_{3} \mathrm{O}_{4}$ nanocomposites prepared via a novel rapid combustion process. Int J Biol Macromol 162:845-852

24. Ji S, Kim S, Song W, Myung S, Lim J, Jung H, Seok K, Lee S (2019) An eco-friendly cellulose acetate chemical sorbent for hazardous volatile organic liquid spill: a perfect material to solve the issue of evaporating hazards. Cellulose 26:2587-2597 
25. Kandiah M, Nilsen M, Usseglio S, Jakobsen S, Olsbye U, Tilset M, Larabi C, Quadrelli E, Bonino F, lillerud K (2010) Synthesis and stability of tagged UiO-66 Zr-MOFs. Chem Mater 22:6632-6640

26. Karumuri S, Hiziroglu S, Kalkan A (2015) Thermoset-cross-linked lignocellulose: A moldable plant biomass. ACS Appl Mater Interfaces 7:6596-6604

27. Khoshnevisan K, Bordbar A, Zare D, Davoodi D, Noruzi M, Barkhi M, Tabatabaei M (2011) Immobilization of cellulase enzyme on superparamagnetic nanoparticles and determination of its activity and stability. Chem Eng J 171:669-673

28. Ladole M, Mevada J, Pandit A (2017) Ultrasonic hyperactivation of cellulase immobilized on magnetic nanoparticles. Bioresour Technol 239:117-126

29. Liang K, Coghlan C, Bell S, Doonan C, Falcaro P (2016) Enzyme encapsulation in zeolitic imidazolate frameworks: A comparison between controlled co-precipitation and biomimetic mineralisation. Chem Commun 52:473-476

30. Liang K, Ricco R, Doherty C, Styles M, Bell S, Kirby N, Mudie S, Haylock D, Hill A, Doonan C (2015) Biomimetic mineralization of metal-organic frameworks as protective coatings for biomacromolecules. Nat Commun 6:1-8

31. Liang K, Wang R, Boutter M, Doherty C, Mulet X, Richardson J (2017) Biomimetic mineralization of metal-organic frameworks around polysaccharides. Chem Commun 53:1249-1252

32. Liu W, Yang N, Chen Y, Lirio S, Wu C, Lin C, Huang H (2015) Lipase-supported metal-organic framework bioreactor catalyzes warfarin synthesis. Chem - A Eur J 21:115-119

33. Lozano P, Bernal B, Jara A, Belleville M (2014) Enzymatic membrane reactor for full saccharification of ionic liquid-pretreated microcrystalline cellulose. Bioresour Technol 151:159-165

34. Lyu F, Zhang Y, Zare R, Ge J, Liu Z (2014) One-pot synthesis of protein-embedded metal-organic frameworks with enhanced biological activities. Nano Lett 14:5761-5765

35. Mehta J, Bhardwaj N, Bhardwaj S, Kim K, Deep A (2016) Recent advances in enzyme immobilization techniques: Metal-organic frameworks as novel substrates. Coord Chem Rev 322:30-40

36. Miller G, Blum R, Glennon W, Burton A (1960) Measurement of carboxymethylcellulase activity. Anal Biochem 1:127-132

37. Phipps J, Chen H, Donovan C, Dominguez D, Beyzavi M (2020) Catalytic Activity, Stability, and Loading Trends of Alcohol Dehydrogenase Enzyme Encapsulated in a Metal-Organic Framework. ACS Appl Mater Interfaces 12:26084-26094

38. Qi B, Luo J, Wan Y (2018) Immobilization of cellulase on a core-shell structured metal-organic framework composites: Better inhibitors tolerance and easier recycling. Bioresour Technol 268:577582

39. Saha K, Verma P, Sikder J, Chakraborty S, Curcio S (2019) Synthesis of chitosan-cellulase nanohybrid and immobilization on alginate beads for hydrolysis of ionic liquid pretreated sugarcane bagasse. Renew Energy 133:66-76 
40. Schaate A, Roy P, Godt A, Lippke J, Waltz F, Wiebcke M, Behrens P (2011) Modulated synthesis of Zrbased metal-organic frameworks: From nano to single crystals. Chem - A Eur J 17:6643-6651

41. Shearer G, Chavan S, Ethiraj J, Vitillo J, Lillerud K (2014) Tuned to perfection: Ironing out the defects in metal-organic framework UiO-66. Chem Mater 26:4068-4071

42. Sing K (1985) Reporting Physisorption Data for Gas/Solid Systems with Special Reference to the Determination of Surface Area and Porosity. Pure Appl Chem 57:603-619

43. Wang J, Wang L, Yu H, Abdin Z, Chen Y, Chen Q, Zhou W, Zhang H, Chen X (2016) Recent progress on synthesis, property and application of modified chitosan: An overview. Int J Biol Macromol 88:333344

44. Wang W, Zhang Q, Sun X, Chen D, Insam H, Koide R, Zhang S (2020) Effects of mixed-species litter on bacterial and fungal lignocellulose degradation functions during litter decomposition. Soil Biol Biochem 141:107690

45. Xu J, He B, Wu B, Wang B, Wang C, Hu L (2014) An ionic liquid tolerant cellulase derived from chemically polluted microhabitats and its application in in situ saccharification of rice straw. Bioresour Technol 157:166-173

46. Zhang J, Koubaa A, Xing D, Wang H, Wang Y, Liu W, Zhang Z, Wang X, Wang Q (2020) Conversion of lignocellulose into biochar and furfural through boron complexation and esterification reactions. Bioresour Technol 312:123586

47. Zhang Q, Lin D, Yao S (2015a) Review on biomedical and bioengineering applications of cellulose sulfate. Carbohydr Polym 132:311-322

48. Zhang W, Qiu J, Feng H, Zang L, Sakai E (2015b) Increase in stability of cellulase immobilized on functionalized magnetic nanospheres. J Magn Magn Mater 375:117-123

49. Zhang Y, Yue Q, Zagho M, Zhang J, Elzatahry A, Jiang Y, Deng Y (2019) Core-Shell Magnetic Mesoporous Silica Microspheres with Large Mesopores for Enzyme Immobilization in Biocatalysis. ACS Appl Mater Interfaces 11:10356-10363

50. Zhou M, Ju X, Zhou Z, Yan L, Li L (2019) Development of an Immobilized Cellulase System Based on Metal-Organic Frameworks for Improving lonic Liquid Tolerance and in Situ Saccharification of Bagasse. ACS Sustain Chem Eng 7:19185-19193

51. Zhou Z, Gao Z, Shen H, Li M, Yang Y (2020) Metal-Organic Framework in Situ Post-Encapsulating DNA-Enzyme Composites on a Magnetic Carrier with High Stability and Reusability. ACS Appl Mater Interfaces 12:7510-7517

52. Zhu C, Fang Z, Su T, Li X, Liu Q (2018) Cellulase immobilized on mesoporous biochar synthesized by ionothermal carbonization of cellulose. Cellulose 25:2473-2485

\section{Figures}


$\mathbf{a}$

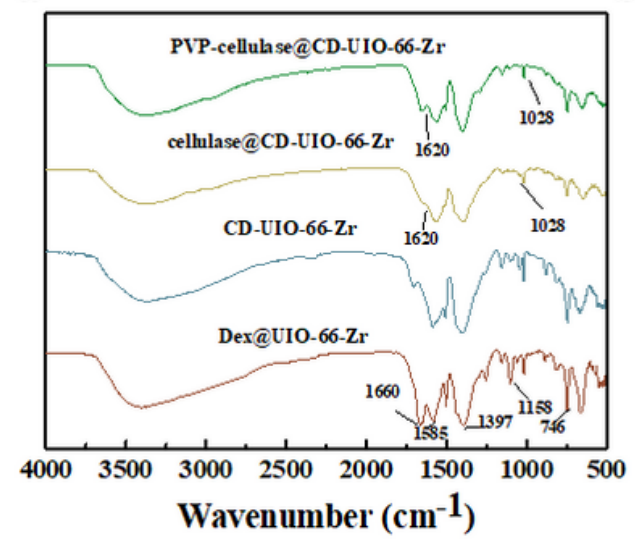

b

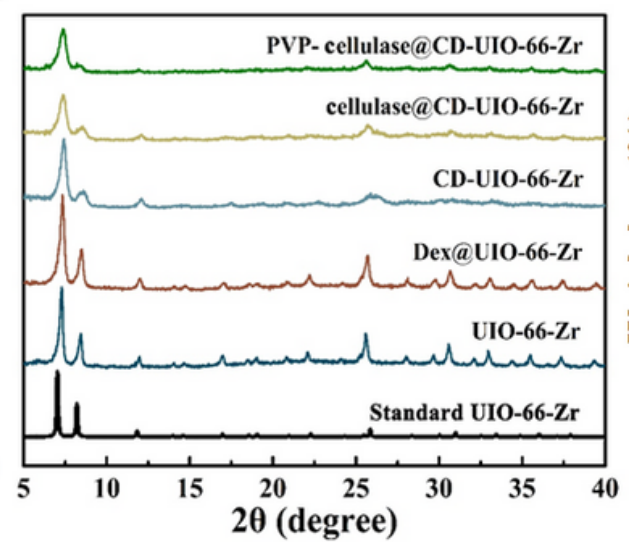

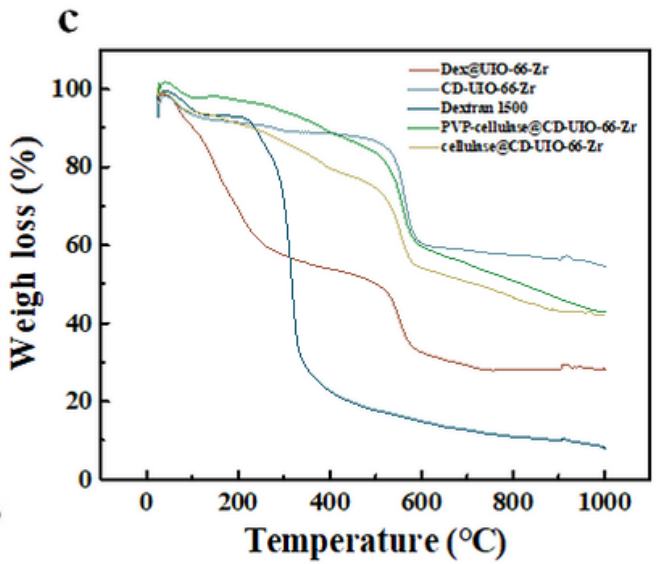

\section{Figure 1}

(a) FT-IR spectra of Dex@UIO-66-Zr, CD-UIO-66-Zr, cellulase@CD-UIO-66-Zr and PVP-cellulase@CD-UIO-66Zr; (b) XRD patterns of UI0-66-Zr, Dex@UIO-66-Zr, CD-UI0-66-Zr, cellulase@CD-UIO-66-Zr and PVPcellulase@CD-UIO-66-Zr; (c) TGA spectrum of Dextran 1500, Dex@UI0-66-Zr, CD-UIO-66-Zr, cellulase@CDUI0-66-Zr and PVP-cellulase@CD-UI0-66-Zr.
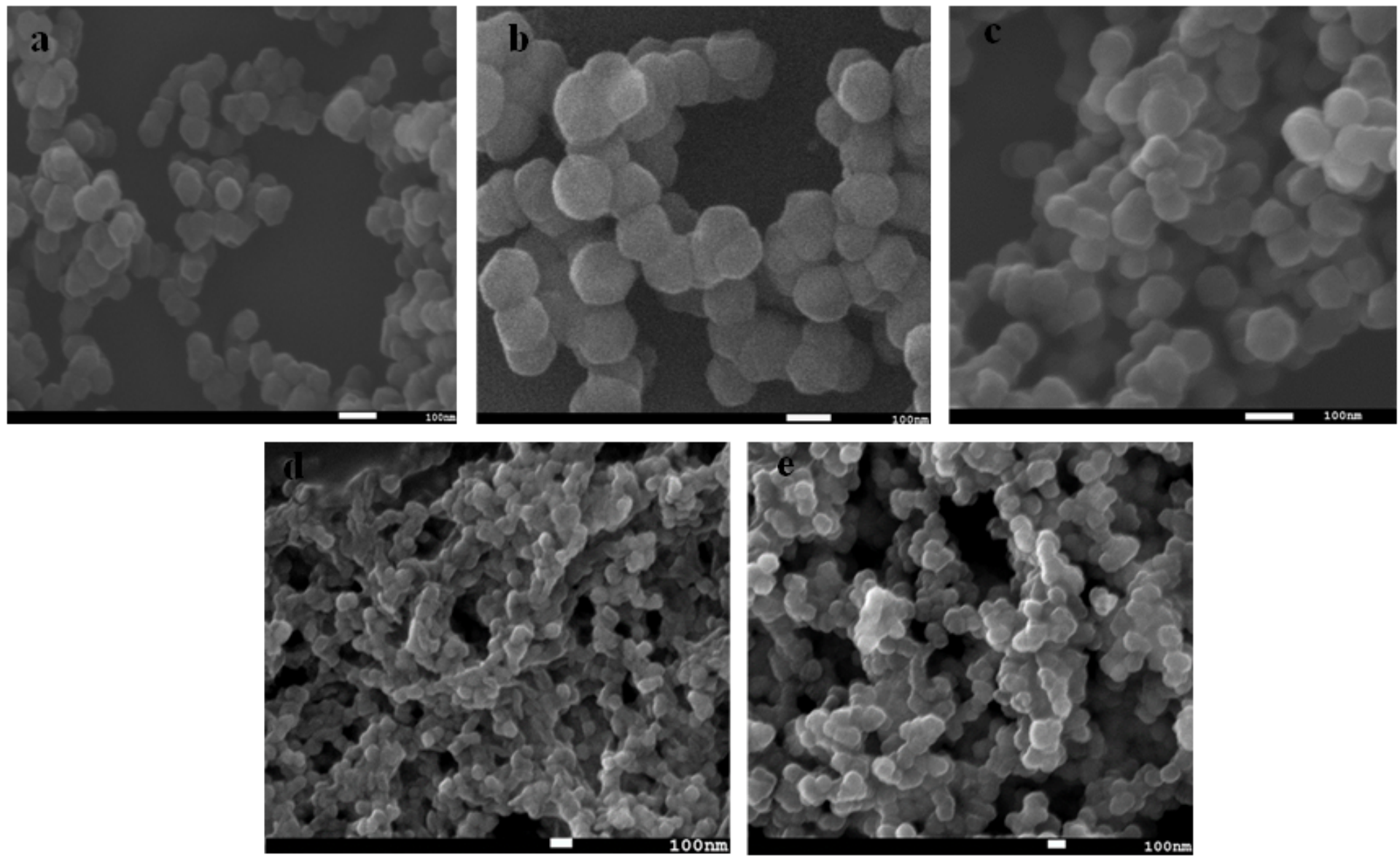

Figure 2

SEM images of UIO-66-Zr (a), Dex@UI0-66-Zr (b), CD-UI0-66-Zr (c), PVP-cellulase@UIO-66-Zr (d), and cellulase@UIO-66-Zr (e) 

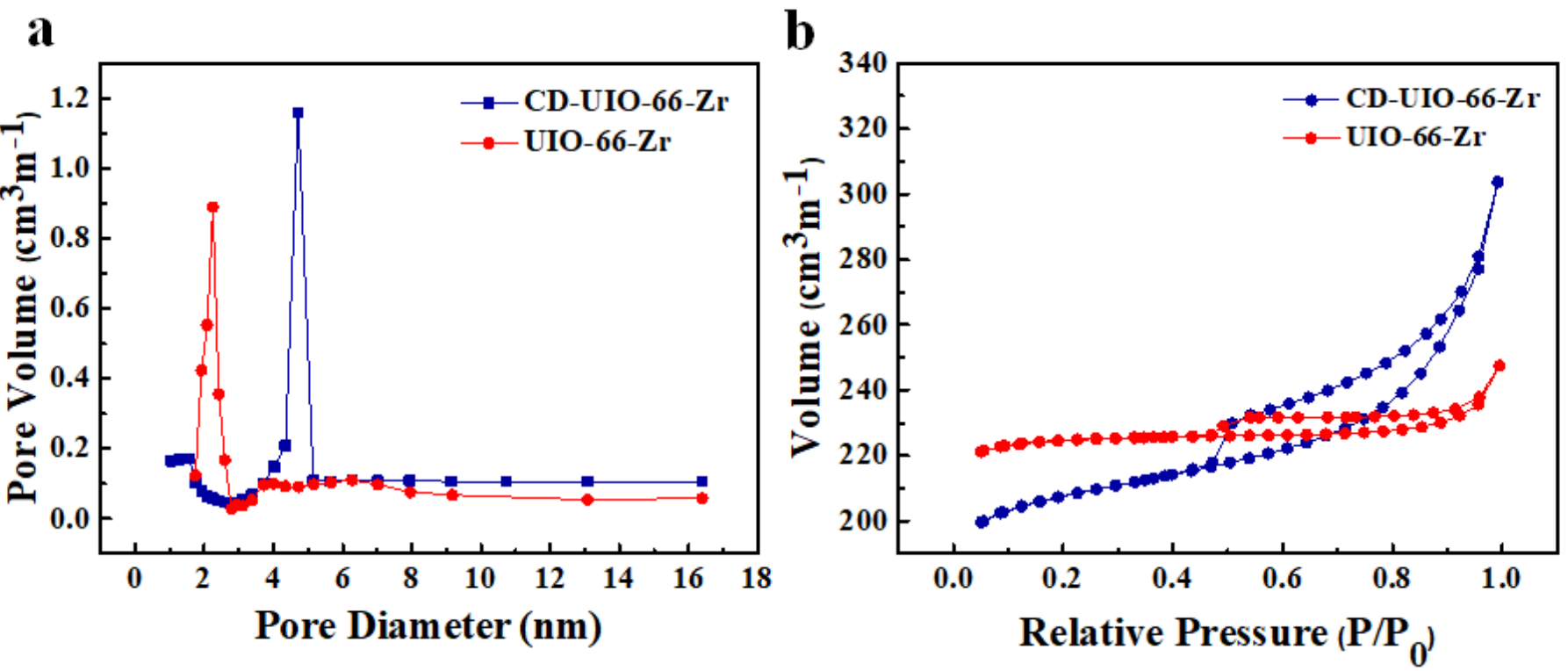

Figure 3

(a) BJH pore diameter distributions of CD-UIO-66-Zr and UIO-66-Zr; (b) BJH N2 adsorption-desorption isotherms of CD-UIO-66-Zr and UIO-66-Zr
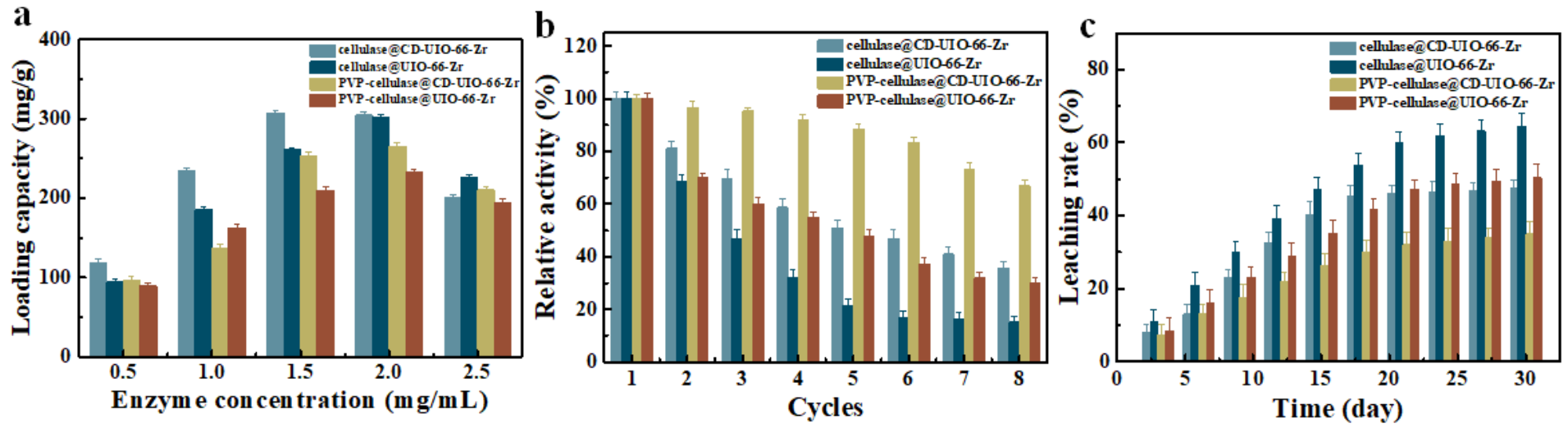

Figure 4

(a) Effect of enzyme concentration on loading capacity of cellulase@CD-UIO-66-Zr, cellulase@UIO-66-Zr, PVP-cellulase@CD-UI0-66-Zr and PVP-cellulase@UI0-66-Zr; (b) Cycling stability; (c) Leaching test of cellulase@UIO-66-Zr, cellulase@CD-UIO-66-Zr, PVP-cellulase@CD-UIO-66-Zr and PVP-cellulase@UIO-66-Zr. 
$\mathbf{a}$

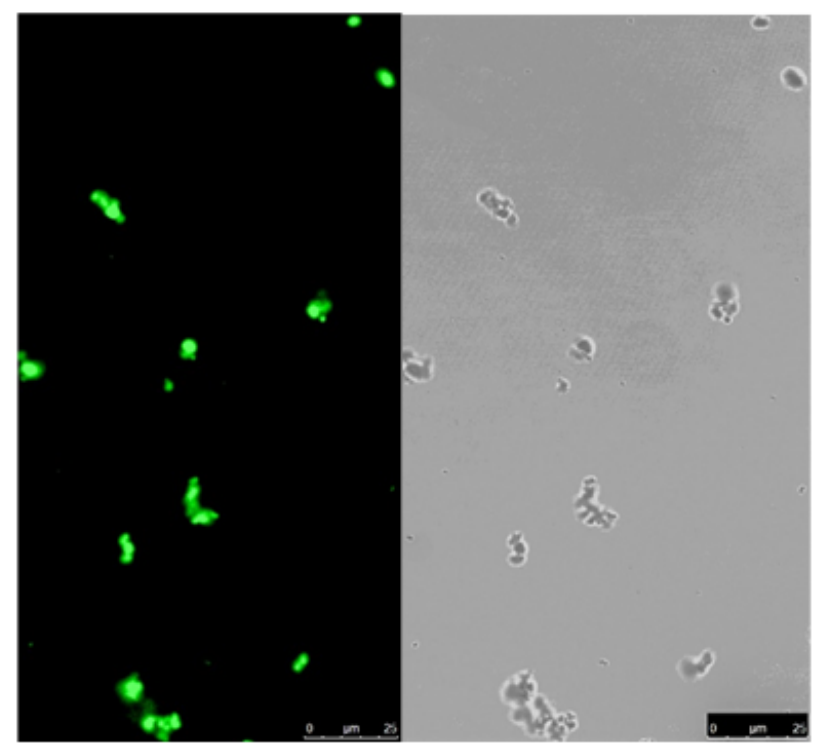

b

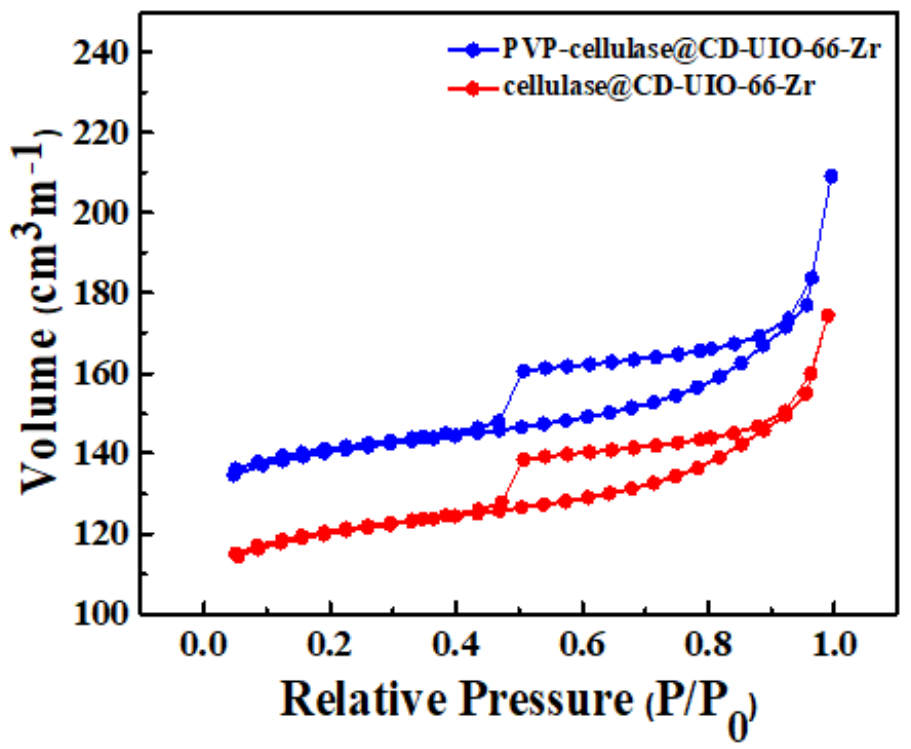

Figure 5

(a) CLSM of FITC-Cellulase@CD-UIO-66-Zr; (b) BJH N2 adsorption-desorption isotherms of PVPcellulase@CD-UIO-66-Zr and cellulase@CD-UIO-66-Zr.

$\mathbf{a}$

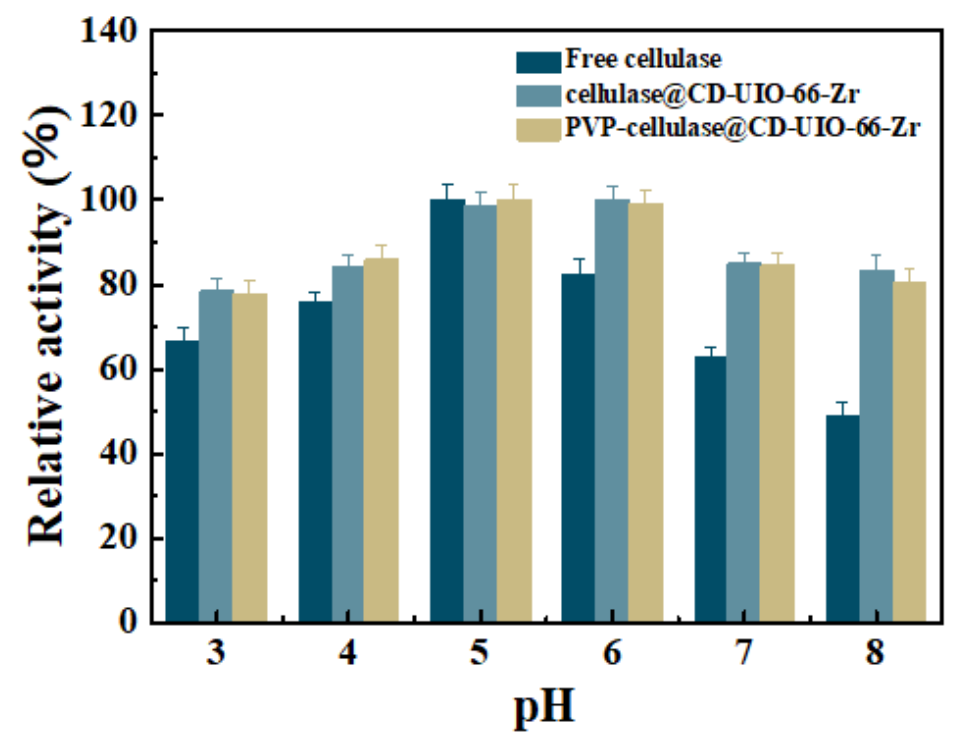

b

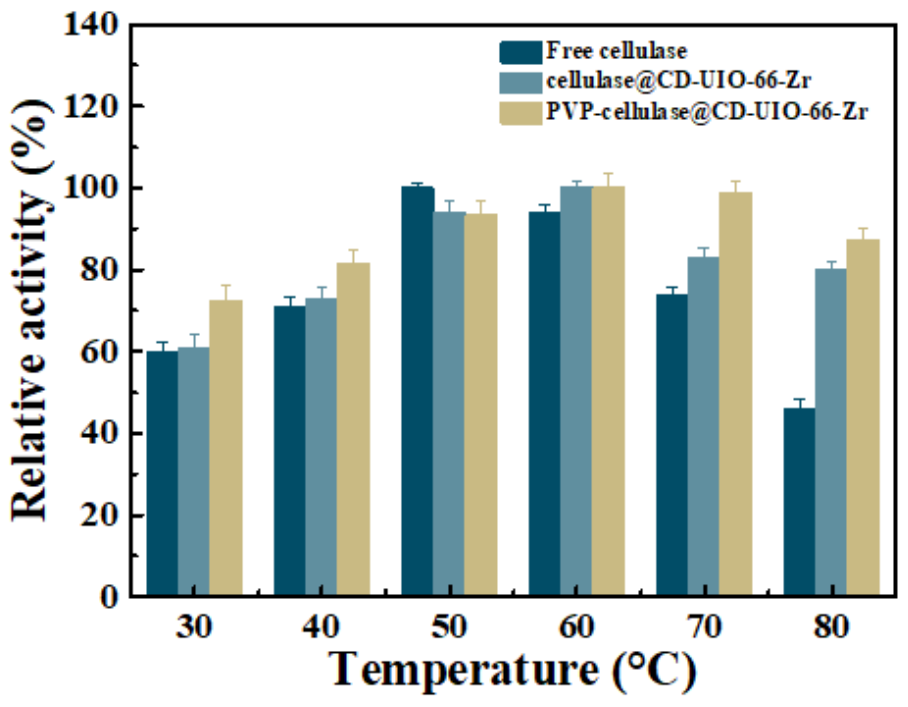

Figure 6

Effects of reaction pH (a) and temperature (b) on catalytic activity of free cellulase, cellulase@CD-UIO-66Zr and PVP-cellulase@CD-UIO-66-Zr. 
a

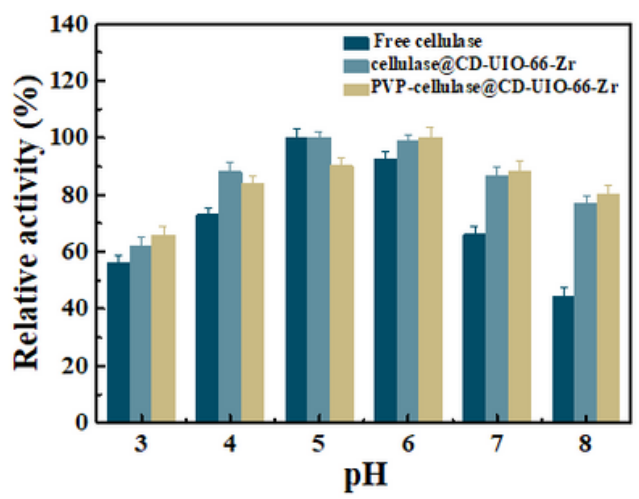

b

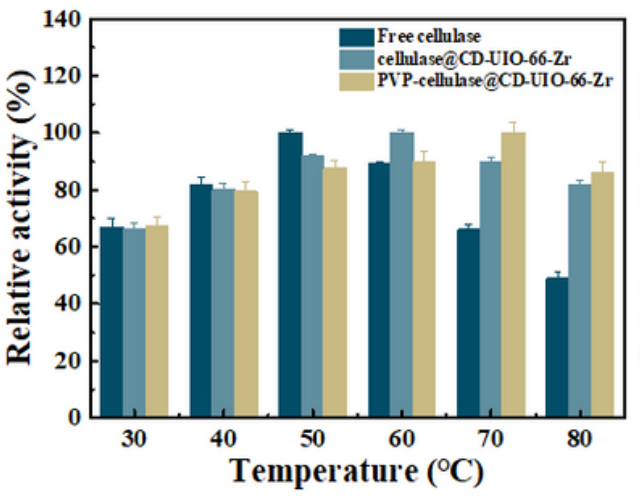

c

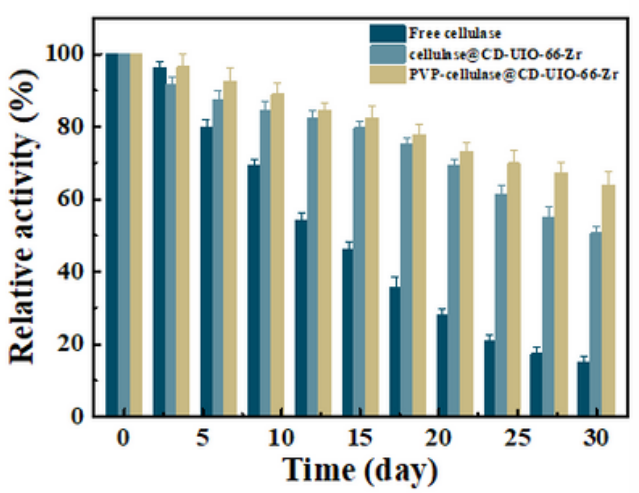

Figure 7

pH stability (a), thermo-stability(b) and storage stability(c) of free cellulase, cellulase@CD-UI0-66-Zr and PVP-cellulase@CD-UIO-66-Zr.

\section{Supplementary Files}

This is a list of supplementary files associated with this preprint. Click to download.

- Scheme1.png

- SI.docx 